\title{
Balance-Mechanisms Governing Equilibrium
}

\author{
By Dr. ANNIE THERON, M.B. Chb. M. Med. \\ Senior Physician of the Neurogical Department-General Hospital, Pretoria. \\ A Lecture given to the Northern Transvaal Branch Members of the South African Society of Physiotherapy, \\ in November, 1959.
}

\begin{abstract}
$\mathbf{O}^{\mathrm{v}}$ VER the past three centuries or more Neurologists have displayed great interest in the underlying nervous mechanisms concerned with the maintenance of posture and equilibrium. The fact that a human can maintain his equilibrium in spite of continuous unrelated movements of his eyes, head, trunk and extremities, is something that is usually accepted without thinking. But actually this phenomenon depends on a very elaborate system of reflexes initiated by a mass of stimuli originating in almost every part of the body, e.g. the eyes, ears, muscles and joints. These impulses are integrated and interpreted by the central nervous system which in its turn sends out messages to all the different skeletal muscles in order that the appropriate movements are carried out and equilibtium is maintained.

It will be necessary to discuss in more detail the different components of this system:-
\end{abstract}

\section{Vestibular Apparatus.}

Each inner ear contains a delicate organ of balance called the labyrinth or vestibulem. It consists of three semi-circular canals, each lying in a different plane and communicating with two further spaces, the sacculus and utriculus. This whole system is contained in a bony labyrinth which is lined with membrane. The spaces are all filled with fluid, the endolymph, which by its movement and gravity pressure stimulates groups of nerve endings situated in the menbranous lining. In this way every postural change or movement of the head is recorded and the impulses are carried along the vistibular nerve to nuclei situated in the brainstem. They are relayed from here to different parts of the nervous system of which the following are the most important:-

(a) The Cerebellum. This forms the main centre of equilibrium regulation.

(b) The Spinal Cord. Here vestibular stimuli have a direct influence on the anterior horn cells and therefore on contraction of skeletal muscles.

(c) Further Vestibular Impulses are conducted upwards in the brainstem to centres governing eye movements. This connection explains nystagmus or the involuntary jerky eye movements so of ten observed in disturbances of the vestibular apparatus.

(d) Another important connection is between the vestibular nuclei and the vital centres concerned with breathing, blood pressure control, pulse-rate and vomiting. Disturbances of these functions e.g. pallor, vomiting, fast pulse and low blood-pressure are clearly seen in attacks of vestibular dysfunction e.g. motion sickness.

\section{The Cerebellum.}

This is the main integrating organ in the regulation of equilibrium. It consists of three components; two hemispheres and a connecting piece called the vermis. Although stimuli causing motor activity do not originate in this part of the brain, it is of the utmost importance in regulating co-ordination of movements. The hemispheres influence voluntary movements e.g. walking and use of the arms. The vermis controls involuntary postural tone, especially in the trunk musculature and patients with lesions of this area show profound loss of equilibrium even in the sitting position.

In order to fulfil all these functions, the cerebellum has to be kept informed about the posture and movement of every part of the body. Impulses from the muscles and joints, so called proprioceptive or kinaesthetic impulses, reach it via the long sensory tracks of the spinal cord; messages from the eyes and ears arrive via brainstem tracks; additional information is sent down from higher cerebral centres. All these stimuli are pooled in the cerebellum where they are continuously sorted and co-ordinated. Suitable regulating impulses are then sent out mainly to the spinal cord and higher motor centres. In this way tone and contraction of every skeletal muscle are modified in such a way that equilibrium is maintained at all times.

\section{Disturbances in Equilibrium.}

The clinical application of all these anatomical and physiological facts can best be illustrated by considering a few clinical diseases which present with disturbances in equilibrium.

The main clinical sign of conditions falling into this category is Ataxia. Ataxia is a condition where in the absence of any muscular weakness, the patient finds it difficult or even impossible to stand still with his feet together, to walk in a straight line and to perform coordinated movements with his arms and hands. Ataxia has many causes. Clinically three main types can be distinguished:-

\section{(a) Sensory Ataxia.}

In these cases the lesion is situated somewhere in the sensory tracks conducting proprioceptive impulses from the trunk and extremities to the cerebellum and other higher centres. Deprived of this source of information the cerebellum is now unable to function normally.

The patient's disability consists of the following: with eyes closed he is unable to determine the position and range of movement of his limbs. He literally does not know where his feet are and without the use of his eyes he is unable to orientate himself in space. He is able to stand still with feet together provided his eyes are open but as soon as he closes them he starts swaying and may fall. He walks on a broad base and has a high steppage gait especially in the dark when visual compensation is impaired. With eyes closed his outstretched arms wander and finger movements lack accuracy. On neurological examination a loss of proprioceptive sensory modalities will be found, mainly the sense of joint position and movement.

Various diseases cause sensory ataxia, e.g.:

(i) Perepheral neuritis, where the long nerves are affected, mainly those to the legs. The conduction of sensory impulses to the spinal cord is impaired and sensory ataxia may result. The condition is further complicated by motor weakness and muscular atrophy due to damage to the motor fibres in the peripheral nerves. Gait disturbance is, therefore, due to two factors:--sensory ataxia and motor weakness, of which any one may predominate. The main causes of peripheral neuritis are vitimin deficiencies, alcoholism, diabetes and various heavy metal poisonings.

(ii) Spinal cord lesions affecting the long sensory tracks are thereby interfering with conduction of proprioceptive impulses, similarly result in sensory ataxia. In this category there are two diseases of importance. 
Tabes Dorsalis: a syphilitic inflammation of the sensory spinal roots and dorsal sensory tracks of the spinal cord. The typical clinical case shows severe ataxia with hypotonia of muscles and very poor co-ordination. Once the disease manifests itself it is progressive in spite of medical treatment and the ultimate prognosis is therefore poor.

Subacute combined degeneration of the cord: this disease is caused by a Vit. B. 12 deficiency and is commonly associated with pernicious anaemia. As in peripheral neuritis the disability is due to both muscular weakness and sensory ataxia. Treatment with Vitamin B. 12 always arrests the disease but certainly does not cure it, especially in advanced cases.

(b) Cerebellar Ataxia.

This type of ataxia becomes evident mostly in chronic bilateral lesions of the cerebellum or its connections. It is seen in its classical form in hereditary or acquired cerebellar degeneration. The hereditary ataxias mostly start in childhood and are slowly progressive. The acquired forms are manifested in late middle life. Both types of cerebellar ataxia carry a poor prognosis. Most patients ultimately become bed-ridden and treatment is purely palliative.

Cerebellar ataxia differs from sensory ataxia in the following respects:

(i) Neurogical examination reveals no sensory deficit the patients are completely aware of the position and range of movement of their limbs.

(ii) Closure of the eyes has little effect on their disability. They sway and sometimes fall whether their eyes are open or closed.

(iii) These patients often have a speech disturbance due to poor co-ordination of the muscles of articulation.

(iv) The inco-ordination of arm movements is often more pronounced than in sensory ataxia. On attempting skilled voluntary movements of the arms they often develop a jerky tremor, the so-called intention tremor.

(v) The disturbance in gait and equilibrium is the outstanding feature. The gait is broadbased; reeling and staggering is more pronounced than in sensory ataxia where visual compensation occurs to some extent.

(c) Vestibular Ataxia.

This type of equilibrium disturbance is caused by a lesion anywhere in the peripheral or central vestibular apparatus, i.e. in the labyrinth itself in the inner ear; along the course of the vestibular nerve; or in its central pathways in the brain-stem; because the labyrinth is a bi-lateral organ, a lesion on one side only causes disturbance of equilibrium only in the acute phase. Very soon the balance is restored by means of compensatory changes on the other side. Therefore these patients characteristically complain of acute attacks of loss of balance which tend to improve within hours or days.

On examination during an attack they are often unable to sit, stand or walk. When the erect posture is assumed they tend to fall sharply to the side of the affected ear. Furthermore, they show the characteristic jerky eye movements, called nystagmus and, very often complain of deafness and ringing noises on the affected side. Their main complaint, however, is that of dizziness or vertigo.

This brings us to one of the most characteristic features of vestibular lesions, i.e. vertigo.

The term "dizziness" is very comprehensive and vague and therefore misleading. It can have a host of meanings: light-headedness; feeling of fainting; nausea; unsteadiness; confused thoughts; and lastly a sense of apparent movement or turning. This last sensation is true vertigo. A full definition of vertigo is as follows: the patient becomes aware of a turning sensation, either of himself or his environment. This hallucination causes him to fall or stagger because his spatial orientation is disturbed.

True vertigo occurs only in vestibular ataxia and not in the cerebellar and sensory types. As already mentioned it is always transitory and never lasts more than a few hours. It is often aggravated or initiated by sudden head movements.

A classical example of acute vestibular disturbance is the disease of Ménière. This condition usually occurs in middle-aged women and is characterised by recurrent attacks of intense vertigo, ataxia leading to complete prostration, nausea, vomiting and frequently deafness and ringing in one ear. The attack is of acute onset, lasts for only a few minutes to half an hour and is usually followed by a period of lightheadedness of gait. Between attacks the patient is quite well except for slight deafness which becomes progressively worse after each attack.

Ménières disease is thought to be due to an acute swelling of the labyrinth with a rise in the fluid pressure leading to abnormal stimulation of the vestibular nerve endings. Very much the same mechanism explains the vestibular ataxia seen during acute ear or mastiod infections.

Lesions of the vestibular nerve and its central pathways within the brainstem also cause vertigo and ataxia but to a lesser extent and seldom lead to the dramatic attacks as described in Ménières disease.

These are then the three main types of ataxiavestibular cerebellar and sensory. Occasionally a similar clinical picture can be seen in lesions of the frontal lobes of the brain and in sufferers from chorea (St. Vitus Dance). In certain forms of hysteria ataxia can be closely stimulated.

\section{THE USE OF PHYSIOTHERAPY IN ATAXIA.}

Physiotherapy is of great value in most of the conditions mentioned. The method used will be determined by the type of ataxia and its prognosis.

As already mentioned the cerebellum has three main sources of information: the labyrinth, the proprioceptive sensory impulses from the muscles and joints and the eyes. In the course of physiotherapy two main principles should be followed:-

1. Damage to a nerve pathway, i.e. nerve endings, a peripheral nerve or a tract in the spinal cord or brainstem, is not always complete or irreversible. By repetitive exercises the remaining nervous tissue can be fully utilised and new pathways may even develop. In addition disuse atrophy of muscles is prevented.

2. The second principle in the treatment of ataxia should be active development of accessory mechanisms. In this way loss of one source of information is compensated for by more efficient functioning of the other sources, e.g. a patient with sensory ataxia should be taught to use his eyes more efficiently in order to gain the information about position and movement which is normally supplied by proprioceptive impulses from the limbs.

\section{Prognosis.}

In order to treat any disease intelligently, a knowledge of its natural course and expected outcome or prognosis is essential. Physiotherapists are notoriously patient and optimistic and often effect miraculous improvement in so called hopeless cases. This is an admirable attitude and it is therefore with trepidation that I even mention the word prognosis.

In general the best results can be expected in sensory ataxia due to peripheral neuritis and early cases of subacute combined degeneration of the spinal cord. In the

(Continued on Page 12). 


\section{SOUTHERN TRANSVAAL BRANCH}

\section{Recent Lectures}

Miss Savin's lecture on Geriatrics was followed up by a tour of the Old Aged Home in Sandringham.

In conjunction with the General Meeting in March, Mr. Neville Cohen, a paraplegic, gave a talk about some of his experiences since his injury. As these included driving his hand-controlled car to England and back again, it proved quite an eye-opener to us. It was also a great stimulus to the three paraplegic patients who were invited from the wards. It would be interesting to hear the impressions of other long term patients regarding hospital life.

Miss Winter gave a stimulating lecture on a controversial subject viz. "Manipulations in the Treatment of Disc Lesions," which left the audience with much food for thought.

\section{Future Lectures}

On May 17th, Miss Jean Blair will talk about her trip to the Physiotherapy Congress in Paris, and all of us who know her are looking forward to this.

The Northern Transvaal Branch secretary, Miss Savin, has extended a very friendly invitation on behalf of her committee for our branch members to attend any of the Society lectures held in Pretoria. Unfortunately we cannot circularize all members about each lecture, but details may be obtained from Miss Schwartz.

We have issued a circular invitation to Northern Transvaal members and we look forward to seeing them at our lectures.

\section{Marriages}

Miss Delyse Dunsden to John Hart in Benoni.

Miss Valery Schwartz to Dr. Rudolph in Johannesburg. Miss J. Pilling, on 5/12/59 to Mr. Reg Van Dyk.

Miss E. Edelinghuys, on 2/4/59 to Dr. Hans Opperman.

\section{Births}

To Mrs. B. H. de Bruin (neé Klooster) on Monday, April 18th, a son, Andries Willem Johannes.

To Mrs. N. van Heerden, on Tuesday, March 17th, a daughter, "Alta."

To Mrs. R. M. Jubson, on Saturday, A pril 23rd, a son.

\section{IN MEMORIUM. \\ MRS. BRONWEN FOURIE, M.C.S.P.}

Trained: at St. Thomas' Hospital, London.

Joined: S.A.S.P., Northern Transvaal Branch, 1952.

I have known Bronnie Fourie (born Vaughan) for the past seven years. She had been away in America for four years, where her husband Brand Fourie, was on the South African ambassadorial staff. As soon as she returned to South Africa she took up her profession as Physiotherapist, and I was indeed most fortunate to obtain her services in charge of my department.

Bronnie Fourie was a born Physiotherapist, for she had the outstanding qualities of humanity, in that she treated all patients, rich and poor white or coloured according to their needs, and with the utmost sympathy and understanding. She was most capable, and even tempered and above all had a sense of humour. She enjoyed her work.

She left South Africa with her husband on his appointment as South African representative to the United Nations, and her only regret on leaving, was that she would not be able to carry on her work as Physiotherapist.

After a year at the United Nations headquarters she returned to South Africa on a short visit to her parents, and it was then she met her tragic end in a motor accident.

The Society of Physiotherapists was deprived of one of its ablest members.

We extend our sincere sympathy to her husband, her parents and the rest of the family, in their untimely and sad bereavement.

H. D. EPSTEIN,
QUESTIONS FOR JOURNAL QUESTION BOX.

1. When is it safe to give movements of nearby joints following a Skin Graft ?

2. Is it true, that cases of Disseminated Sclerosis are rarely found in South Africa, and only in people who have already developed it overseas?

3 . Is it really necessary to remove clothing, when applying SHORT-WA VE Diathermy? Many Physiotherapists in this country disregard this precaution, stating that they have never burnt a patient.

4. What type of Physiotherapy has been found to be most effective in treating hand injuries.

5. Should the grading of muscle power be standardised throughout South Africa.

These questions will be answered by Specialists in each field but in the meantime members are invited to send in their own ideas and suggestions for discussion.

\section{BOOK REVIEW}

\section{Practical Electrotherapy for Physiotherapists By Brenda Savage.}

Published by: Faber \& Faber, 24 Russel Square, London, W.C.I.

$$
\text { Price 30/- net. }
$$

This book gives a short introductory chapter on some physical and mechanical principles of various current forms and pieces of electrical apparatus. In this chapter it also deals with the role played by the skin in the application of different electrical treatments.

It deals further with the physiological effects on the body produced by different forms of electrical and radiation treatments. It also describes in detail the technique of some basic forms of treatments by Electrotherapy, i.e. the applications of Direct Current, certain ionisation treatments as Histamine and Renotin ionisation, the distribution of electrical fields in High Frequency current treatments, the application of S.W.D. coils, and the technique of giving General and Local U.V.R.

And finally and mainly it deals with a vast number of conditions that can be treated by means of Electrotherapy. In this part of the book different forms of treatment are discussed, some of the more specialised ones being described in detail.

The book is illustrated by a great number of very clear and valuable diagrams.

This book is a must in any Physiotherapy library. It is one of very few books attempting to give a detailed account on techniques and possibilities in Electrotherapy, something sadly lacking up to now as contrasted with the vast number of books being written and published on the electrical and mechanical part of Electrotherapy apparatus.

We are sure that every physiotherapist and physiotherapy student will be very grateful indeed to be able to acquire a book that deals with the practical application of treatments by Electrotherapy.

\section{BALANCE (Continued from Page 7).}

abovementioned cases vitamin therapy combined with intensive physiotherapy will lead to a cure in most of them. The results in tabes dorsalis are unfortunately less encouraging, because damage to the nervous system is usually irreversible. Nevertheless graduated exercises with special attention to the development of other sensory mechanisms, especially the eyesight, very often have very gratifying results.

I believe special physiotherapeutic methods have been devised in the treatment of vestibular disturbances. Of these I have no experience.

The ultimate outcome in cerebellar ataxia is that of a bed-ridden patient, but physiotherapy can postpone this considerably and give the patient many years of useful life. 